\title{
NKT cells mediate the recruitment of neutrophils by stimulating epithelial chemokine secretion during colitis
}

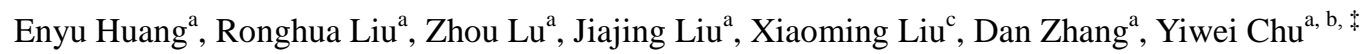

${ }^{\text {a }}$ Department of Immunology and Key Laboratory of Medical Molecular Virology of MOE/MOH, School of Basic Medical Sciences, Fudan University. Shanghai, 200032, People's Republic of China.

${ }^{\mathrm{b}}$ Biotherapy Research Center, Fudan University. Shanghai 200032, China.

${ }^{\mathrm{c}}$ Department of Dermatology, Shenzhen Hospital, Peking University, Shenzhen, Guangdong 518036, China.

+ Correspondence to: Yiwei Chu, Department of Immunology, School of Basic Medical Sciences, Fudan University, No.138, Yi Xue Yuan Rd., mail box 226, Shanghai, 200032, People's Republic of China, 200032, Tel: +86-21-54237324,

E-mail: yiweichu@ fudan.edu.cn, Fax: +86-21-54237324 


\section{Abstract:}

Ulcerative colitis (UC) is a kind of inflammatory bowel diseases characterized by chronic inflammation and ulcer in colon, and UC patients have increased risk of getting colorectal cancer. NKT cells are cells that express both NK cell markers and semi-invariant CD1d-restricted TCRs, can regulate immune responses via secreting a variety of cytokines upon activation. In our research, we found that the NKT cell-deficient CD1 $\mathrm{d}^{-/-}$mice had relieved colitis in the DSS-induced colitis model. Further investigations revealed that the colon of $\mathrm{CD}^{-\mathrm{d}^{--}}$mice expressed less neutrophil-attracting chemokine CXCL 1, 2 and 3, and had decreased neutrophil infiltration. Infiltrated neutrophils also produced less reactive oxygen species (ROS) and TNF- $\alpha$, indicating they may cause less epithelial damage. In addition, colitis-associated colorectal cancer was also relieved in $\mathrm{CD}^{-\mathrm{d}^{--}}$mice. During colitis, NKT cells strongly expressed TNF- $\alpha$, which could stimulate CXCL 1, 2, 3 expressions by the epithelium. In conclusion, NKT cells can regulate colitis via the NKT cell-epithelium-neutrophil axis. Targeting this mechanism may help to improve the therapy of UC and prevent colitis-associated colorectal cancer.

Keywords: inflammatory bowel disease; natural killer T cell; neutrophil; colitis-associated colorectal cancer

\section{Introduction}

Ulcerative colitis (UC) is a type of inflammatory bowel diseases (IBD) which is usually confined to the colon and the rectum, with diffuse and superficial lesions of the mucosa. UC generally occurs in young adulthood and it's incurable, continues throughout one's lifetime. The course of the disease is usually repetitive flare and remission, and UC patients have increased colon cancer incidence. UC incidence has increased over the past 50 years [1,2]. NKT cells are innate-like lymphocytes that express semi-invariant $\alpha \beta T C R$ and NK cell lineage markers (NK1.1 in C57BL/6 mice and NK161 in human). NKT cells are mainly divided into two subtypes. Type I NKT cells, also called invariant NKT cells (iNKT cells), express the invariant $\alpha \beta T C R s$ (V $\alpha 14-\mathrm{J} \alpha 18$ coupled with V $\beta 8.2, \mathrm{~V} \beta 7$ or V $\beta 2$ in 
mice, V $\alpha 24-J \alpha 18$ coupled with V $\beta 11$ in human), while type II NKT cells' TCRs are comparatively more various, but still limited. Both subtypes recognize glycolipid or glycerol lipid antigens presented via CD1d, and mice without CD1d fail to produce NKT cells due to compromised positive selection in the thymus [3]. NKT cells mainly function by secreting a variety of cytokines to mediate immune responses.

There have been some researches on NKT cells and colitis, and the role of NKT cells may differ according to species and animal models. Fuss et al. reported in UC patients, a type of NKT cells could produce IL-13, which could strengthen the cytotoxicity of $\mathrm{CD} 4^{+} \mathrm{CD} 161^{+}$cells to HT-29 colon cancer cell-line [4]. Further experiments showed $36 \%$ of lamina propria mononuclear cells (LPMCs) from UC patients are lyso-sulfatide-responsive NKT cells, and secrete IL-13 upon activation [5]. Consistently, in mouse models, Heller et al. reported that IL-13 and NKT cells were important for the development of Oxazolone-induced colitis [6], and Camelo et al. further revealed CD1d-reactive NKT cells were one source of IL-13 [7]. However, Liao et al. reported although mice with a subset of NKT cells (expressing V $\alpha 3.2 \mathrm{~V} \beta 9$ TCRs) over-produced seemed normal, in mice with both V $\alpha 3.2 \mathrm{~V} \beta 9$ TCR and CD1d over-expressed, who had decreased $\mathrm{V} \alpha 3.2^{+} \mathrm{V} \beta 9^{+} \mathrm{NKT}$ cells due to strengthened negative selection, IBD was spontaneously developed, indicating NKT cells may naturally have a protective role and their dysregulation can induce colitis [8]. Moreover, Kim et al. reported in DSS-induced colitis model, NKT cells could secrete IL-9 and protect mice from colitis by suppressing IFN- $\gamma$ and IL-17A expression in colon [9], and Yoshioka, et al.'s research also suggested the protective role of NKT cells in colitis and colon cancer [10].

Researches above showed that the role of NKT cells during colitis is complicated, and they mostly focused on their helper T cell-like function. However, researches of their influence on another aspect of immune response which is also very important in colitis, the innate immune response, are still limited.

Neutrophils are one of crucial innate immune cells, and their infiltration is always identified as a hallmark of various inflammatory diseases, including colitis. Neutrophils play a critical role in the maintenance of intestinal homeostasis, while excessive recruitment and accumulation of activated neutrophils in the intestine under pathological conditions 
such as colitis is associated with mucosal injury [11].The research on NKT cells and neutrophils during colitis is still limited, and their relationship is more investigated in hepatitis, renal ischemia-reperfusion injury (IRI) and pneumonia. The roles of NKT cells and their cytokines are dependent on the disease. They were reported to contribute to chronic-plus-binge ethanol-mediated liver injury [12] and IRI [13], and eliminating or blocking them ameliorated the injury and decreased neutrophil infiltration. Wang et al. showed that NKT cells can secrete IL-4 to recruit neutrophils in liver, and subsequently increase their IFN- $\gamma$ expression to regulate this process [14], and the latter part was confirmed in cholestatic liver injury [15]. However, IL-4 secreted by NKT cells has also been reported to suppress neutrophils during hypersensitivity pneumonitis [16], and IFN- $\gamma$ seem to contribute to the recruitment of neutrophils during Streptococcus pneumonia, possibly by up-regulating the CXCL2 in the lung [17].

In our research, we investigated the role of NKT cells in neutrophil recruitment during colitis. Our findings suggest that NKT cells may recruit neutrophils by promoting the chemokine secretion of epithelial cells. We also observed that neutrophils are more activated in wild type compared with NKT-cell deficient mice. This research may reveal an aspect of the progression of colitis.

\section{Material and Methods:}

\subsection{Mice}

$\mathrm{CD}_{1} \mathrm{~d}^{-/}$mice $(\mathrm{B} 6(\mathrm{C})-\mathrm{CD} 1 \mathrm{~d}<\mathrm{tm} 1.2 \mathrm{Aben}>/ \mathrm{J})$ were purchased from Jackson Laboratory and bred in specific pathogen-free conditions in the Department of Laboratory Animal Science, Fudan University. Wild type mice (C57BL/6) were purchased from the Department of Laboratory Animal Science, Fudan University. All mice used in the experiments were 8-12 weeks old. All animal experiments comply with the National Institutes of Health guide for the care and use of Laboratory animals (NIH Publications No. 8023, revised in 1978).

\subsection{Media and Regents}

Antibodies used were FITC-conjugated anti-mouse TCR- $\beta$ (H57-597) and CD45 (30-F11); PE-conjugated 
anti-mouse NK1.1 (PK136), CD11b (M1/70) and EpCAM (G8.8); APC-conjugated anti-mouse CD3\& (145-2C11), Gr-1 (RB6-8C5) and TNF- $\alpha$ (MP6-XT22); eFluor 450-conjugated anti-mouse CD45 (30-F11) and Gr-1 (RB6-8C5); PE-cy7-conjugated anti-mouse F4/80 (BM8); Alexa-Fluor 488-conjugated anti-mouse CD11c (N418) purchased from ebioscience; and Brilliant Violet 421-conjugated anti-mouse TNF- $\alpha$ (MP6-XT22), PE-cy7-conjugated anti-mouse CD45 (30-F11), Alexa-Fluor 647-conjugated anti-mouse CD1d (1B1) purchased from BioLegend. APC-conjugated Rat IgG1 $\kappa($ BRG1, ebioscience) and Brilliant Violet 421-conjugated rat IgG1 $\kappa$ (RTK2071, Biolegend) were used as isotype controls when staining for cytokines.

\subsection{The mouse model for colitis and colitis-associated colorectal cancer}

To induce colitis, Dextran sulfate sodium salt (DSS, MP Biomedicals) was dissolved in drinking water to $2.5 \%$ and treated the mice for 6 days. To establish the model for colitis-associated colorectal cancer, mice were injected with $8 \mu \mathrm{g} / \mathrm{g}$ of Azoxymethane (AOM, Sigma-Aldrich) intraperitoneally (for negative control, with $0.9 \% \mathrm{NaCl}$ solution). After 5 days, they were treated with 2.5\% DSS for 6 days, then with normal water for 14 days. The DSS-normal water cycle was performed for another two rounds and the mice were sacrificed 44 days after the third withdrawal of DSS.

\subsection{Isolation of cells from the colon}

Colons were excised out and opened longitudinally, with fats and fecal removed. After scraping away the mucus, they were cut into $0.5 \mathrm{~cm}$ long pieces and shaken in $25 \mathrm{ml}$ of CMF buffer for $60 \mathrm{~min}$, at $37{ }^{\circ} \mathrm{C}, 260 \mathrm{rpm}$, to isolate epithelial cells. CMF buffer is D-Hank's buffer (Genom) containing 2\% of FBS (Gemini), $10.9 \mathrm{mM}$ of HEPES, 1mM of DTT, $1 \mathrm{mM}$ of EDTA and $25 \mathrm{mM}$ of Sodium hydrogen carbonate (Sigma-Aldrich). The colons were filtered with $70 \mu \mathrm{m}$ nylon cell strainer (Corning) and the epithelial cells were collected by centrifuging every $30 \mathrm{~min}$. Epithelial cells were lysed with RNAiso Plus (TAKARA) and stored at $-80{ }^{\circ} \mathrm{C}$. The lamina propria (LP) were cut into $1 \mathrm{~mm}$ long fragments, put in $5 \mathrm{ml}$ of RPMI 1640 Medium (Life Technologies) containing 10\% FBS, $1 \mathrm{mg} / \mathrm{ml}$ of 
Collagenase D (Roche) and $0.08 \mathrm{mg} / \mathrm{ml}$ of DNase I (Sigma-Aldrich) and shaken for $60 \mathrm{~min}, 260 \mathrm{rpm}, 37{ }^{\circ} \mathrm{C}$ 。Then the cells were filtered and collected.

\subsection{Stimulation of epithelial cells with TNF- $\alpha$}

Colons from wild type mice were cut into $0.5 \mathrm{~cm}$ pieces and incubated in PBS containing $2 \mathrm{mM}$ of EDTA and 0.2 mM DTT for $30 \mathrm{~min}$, at $4{ }^{\circ} \mathrm{C}$. After that, they were pipetted vigorously to isolate epithelial cells. After centrifuging and removal of the supernatant, the cells were seeded in a 6-well plate ( Thermo Scientific) precoated with Geltrex (Life Technologies) and incubated in DMEM (GE Healthcare Life Sciences) containing 10\% of FBS, 100U/ml of Penicillin, $0.1 \mathrm{mg} / \mathrm{ml}$ of Streptomycin, $2.5 \mu \mathrm{g} / \mathrm{ml}$ of fungizone (all from Sangon Biotech) and indicated concentrations of murin TNF- $\alpha$ (Peprotech) at $37{ }^{\circ} \mathrm{C}$ in $5 \% \mathrm{CO}_{2}$, at a concentration of $1.5^{*} 10^{6} \mathrm{cells} / 4 \mathrm{ml} / \mathrm{well}$. Twenty-four or forty-eight hours later, the cells were collected and lysed with RNAiso.

\subsection{Realtime PCR}

RNA from epithelial cells was extracted according to manufacturer's protocol. One microgram of RNA was reverse transcribed using PrimeScript ${ }^{\mathrm{TM}}$ RT reagent Kit with gDNA Eraser (Perfect Real Time) and gene expression was detected using SYBR ${ }^{\circledR}$ Premix Ex Taq ${ }^{\mathrm{TM}}$ II (Tli RNaseH Plus) (purchased from TAKARA). Primers used are synthesized by Sangon Biotech. CXCL1: Sense Primer (S): CCAGTTCCAGCACTCCAGACTC, Antisense Primer (A): GCAGCTCATTGGCGATAGGC. CXCL2: (S): CCAACCACCAGGCTACAGG, (A): GCGTCACACTCAAGCTCTG. CXCL3: (S): CGCTGTCAGTGCCTGAACACCCTAC, (A): ACCATCCTTGAGAGTGGCTATGACTTCTGT; CXCL5: (S): GCCCTACGGTGGAAGTCATA, (A): GTGCATTCCGCTTAGCTTTC. Cyclin D1: (S): CTGAACCTGGGCAGCCCCAA, (A): GGCGCAGGCTTGACTCCAGA. Beta-actin: (S): CTGTCGAGTCGCGTCCACC, (A): CATGCCGGAGCCGTTGTCG. beta-catenin:(S):TCGTGCTGGTGACAGGGAAGAC, (A):ATGAAGGCGAACGGCATTCTGG. 


\subsection{Flow-cytometry}

Cells were stained by fluorchrome-conjugated antibodies according to manufacturer's protocols. For the intracellular staining of TNF- $\alpha$ in NKT cells, cells were stimulated by incubating in RPMI 1640 medium containing $10 \%$ of FBS, $55 \mathrm{nM}$ of 2-Mercaptoethanol (Life Technologies), $0.5 \mu \mathrm{g} / \mathrm{ml}$ of Ionomycin (Peprotech), $0.5 \mu \mathrm{g} / \mathrm{ml}$ of Phorbol 12-myristate 13-acetate (PMA, Sigma-Aldrich), and $2 \mu \mathrm{M}$ of Monensin (ebioscience); for intracellular staining of TNF- $\alpha$ in neutrophils, cells were stimulated by incubating in RPMI 1640 medium containing $10 \%$ of FBS, 10ng/ml of LPS (Sigma-Aldrich) and $2 \mu \mathrm{M}$ of Monensin. All stimulated at a concentration of $2 \times 10^{6}$ cells $/ 2 \mathrm{ml} / \mathrm{well}$, for 5 hours at $37^{\circ} \mathrm{C}$, in $5 \% \mathrm{CO}_{2}$. After stimulation, the cells were stained for surface markers and cytokines with Intracellular Fixation and Permeabilization Buffer Set (ebioscience) according to manufacturer's protocol.

\subsection{ROS detection in neutrophils}

To detect the production of reactive oxygen species (ROS) within neutrophils, after surface staining, cells were incubated in PBS containing 10 $\mu \mathrm{M}$ of 2', 7'- Dichlorofluorescin diacetate (DCFH-DA, Sigma-Aldrich), in $37{ }^{\circ} \mathrm{C}$ for $30 \mathrm{~min}$, and were washed and analyzed. The cells were detected using $488 \mathrm{~nm}$ laser and the mean fluorescence intensity (MFI) of FL1 (530/40 bandpass) represented the amount of ROS produced by neutrophils.

\subsection{Statistics}

GraphPad Prism 5 was used to analyze the data. Values were shown as mean \pm SEM. Unpaired $t$ test was performed and $\mathrm{p}<0.05$ was regarded as significant.

\section{Results:}

3.1 DSS-induced colitis was relieved in $\mathrm{CD}^{-\mathrm{d}^{-/-}}$mice.

We traced the body weight of mice during DSS treatment. Although we used mice of the same age, the body weights still vary, so we used relative body weight, defined as the current body weight divided by that on Day 0 , to indicate weight change. We found that compared to wild type mice, $\mathrm{CD} 1 \mathrm{~d}^{-{ }^{-}}$mice lost weight slower and the total weight loss 
on Day 6 was ameliorated. (Fig. 1 A \& B) During colitis, the spleens are usually swollen and infiltrated by neutrophils, which is a symbol of systemic inflammation. $\underline{\mathrm{CD}_{\mathrm{d}} \mathrm{d}^{--} \text {mice also had relieved systemic inflammation, }}$ with relieved splenomegaly (Fig. 1 C), less CD11b ${ }^{+}$Gr- $1^{\text {hi }}$ neutrophil infiltration (Fig. 1 D) and decreased pro-inflammatory TNF- $\alpha$ secretion of neutrophils upon stimulation. (Fig. 1 E) We confirmed the identities of these cells in the spleen and the colon lamina propria using May-Grünwald-Giemsa staining. Nearly all of these cells in our model showed neutrophil morphology and are true neutrophils. (Fig. S1) In the histological sections of colons stained by hematoxylin \& eosin, we observed $\mathrm{CD} 1 \mathrm{~d}^{-/-}$mice had less leukocyte infiltration in the lamina propria, and the epithelium was less damaged. (Fig. 1 F) The results above indicate that when CD1d was knocked out in mice, colitis was relieved.

\subsection{The neutrophils were less potential to cause damage in lamina propria in CD1 $\mathrm{d}^{-/-}$mice}

Then we investigated the inflammation status of the colon. We found $C D 1 \mathrm{~d}^{--}$mice had significantly less neutrophil infiltration in the lamina propria (LP). (Fig. 2 A) These neutrophils were also less potent to secrete TNF- $\alpha$, which has been reported to induce epithelium apoptosis [18]. (Fig. 2 B) It's widely known that neutrophils can kill pathogens by producing reactive oxygen species (ROS), however, ROS can also cause colon cancer through introducing DNA instability [19, 20], so we used DCFH-DA to detect the production of ROS of neutrophils in LP [21]. We found that neutrophils in $\mathrm{CD}_{1} \mathrm{~d}^{-/-}$mice produced less ROS comparing to wild type mice (Fig. $2 \mathrm{C}$ ). The decreased amount and weakened ability to produce pro-inflammatory cytokine and ROS indicated that the neutrophils were less potential to cause damage in the colons of $\mathrm{CD}_{1} \mathrm{~d}^{-\sigma_{-}}$mice. Besides the effector molecules, the neutrophils in $\mathrm{CD} 1 \mathrm{~d}^{-{ }^{-}}$mice also expressed weaker CXCR2, a common receptor for the neutrophil-attracting chemokines CXCL 1, 2, 3 and 5 (Fig. 2 D). To further investigate the reason why the neutrophils accumulated less in $\mathrm{CD} 1 \mathrm{~d}^{--}$mice, we surmised that the neutrophil-attracting chemkines were down-regulated in $\mathrm{CD}_{1} \mathrm{~d}^{-/}$mice. Because epithelial cells can be activated to secrete a variety of chemokines during inflammation [22], we investigated the expression of chemokines by colon epithelial cells. We found that CXCL 1, 2, 3 and 5 were dramatically decreased in CD1 $\mathrm{d}^{-/-}$mice during colitis, 
compared to wild type mice. (Fig 2. E) The down-regulated CXCL1, 2, 3 and 5 and the decreased CXCR2 on neutrophils may explain why neutrophils accumulated less in $\mathrm{CD}^{-\mathrm{d}^{-/}}$mice during colitis.

3.3 Natural killer T cells strongly produced TNF- $\alpha$ during colitis, which stimulated the chemokine expression of epithelial cells

Because the knockout of CD1d affects NKT cells, we next investigated the activity of NKT cells. Because both subtypes of NKT cells are deleted in CD1d ${ }^{-/-}$mice, we used TCR $\beta$ and NK1.1 as markers for general NKT cells [23]. We found during colitis, NKT cells significantly produced more TNF- $\alpha$. (Fig 3. A \& B) These NKT cells may be stimulated by the lipid antigens presented by dendritic cells, macrophages or epithelial cells in the colon because we detected that both dendritic cells and macrophages in the lamina propria could strongly express CD1d and epithelial cells although at a moderate level, can up-regulate their CD1d during colitis (data not shown). To investigate whether TNF- $\alpha$ can cause the up-regulation of neutrophil-attracting chemokines in epithelial cells, colon epithelial cells from wild type mice were isolated and stimulated with different concentrations of murin TNF- $\alpha$ for 24 or 48 hours. After that, the CXCL expressions of epithelial cells were analyzed using qPCR. We observed that upon $50 \mathrm{ng} / \mathrm{ml}$ of TNF- $\alpha$ stimulation for 24 hours, CXCL1, 2, 3 could be up-regulated. (CXCL5 expression was not affected and data are not shown.) (Fig 3. C) So the accumulation of neutrophils during colitis may be indirectly affected by NKT cells.

\subsection{Colitis-associated colorectal cancer was relieved in $\mathrm{CD}^{-\mathrm{d}^{--}}$mice}

Combined with the carcinogen AOM, repeated DSS treatment can lead to colon cancer, simulating the transformation of chronic UC to colon cancer, in which the pro-inflammatory factors and oxidative stress play an important role [24]. In the colitis-associated colorectal cancer model (Fig 4. A), we observed that comparing to wild type mice, $\mathrm{CD} 1 \mathrm{~d}^{-/-}$mice had obviously fewer and smaller colon tumors (Fig 4. B), and accordingly, colons expressed less cyclin D1 and beta-catenin (Fig 4. C), which had been reported to be important to the oncogenesis and the 
progression of colitis-related colon cancer [25]. These results indicate that CD1d and NKT cells also affected the progression of colon cancer, and the relieved inflammation may be a causative factor.

\section{Discussion:}

From the results above, we propose a hypothesis which may partly explain how NKT cells are involved in colitis. In this hypothesis, NKT cells are activated by lipid antigens presented via CD1d by DCs, macrophages or epithelial cells. These antigens can be exogenous [26], from invaded pathogens, or endogenous, synthesized by activated antigen presenting cells [27]. Activated NKT cells strongly produce cytokines to mediate immune responses, including TNF- $\alpha$. TNF- $\alpha$ stimulates the colon epithelial cells, and triggers the secretion of CXCL 1, 2, 3, which are known neutrophil chemo-attractants. Another neutrophil attracting factor, CXCL5, also has been detected to be differentially expressed between wild type and $\mathrm{CD} 1 \mathrm{~d}^{---}$mice, is possibly influenced by other factors produced by NKT cells. Our research didn’t rule out the possibility that other cytokines may synergize with TNF- $\alpha$ to induce CXCL secretion. NKT cells can affect the amount of neutrophils attracted to the colon, and may also affect their activation status [13]. We can see in CD1 ${ }^{-/-}$mice, neutrophils had decreased pro-inflammatory cytokine TNF- $\alpha$ and ROS production, and lower CXCR2 expression. The decreased chemokine and down-regulated receptor can explain the decreased neutrophils infiltration in CD1 ${ }^{-/-}$mice during colitis. Because TNF- $\alpha$ can induce the production of ROS [19], and the blockade of its major receptor TNF-Rp55 relieved both colitis and colon carcinoma [28], it is possible that NKT cells mainly act as an initiator, attract neutrophils indirectly, and may synergize with other factors to activate neutrophils, making them stay (CXCR2 up-regulation) and become tissue-damaging (TNF- $\alpha$ and ROS up-regulation). After that, the inflammation can be self-sustained, for instance, the ROS production can be sustained by neutrophils' own TNF- $\alpha$. Because the introduction of mutation by ROS and the angiogenesis activity of TNF- $\alpha$ [29] can promote carcinogenesis and tumor growth, abated damage effect of neutrophils in CD1 ${ }^{-/-}$mice can partly explain the reason of reduced carcinogenesis and tumor size in the AOM-DSS model. One defect of our research is 
$\underline{\text { the failure to indicate which subtype of NKT cells is the culprit, however, given that it has been reported in the }}$

DSS-induced colitis model, iNKT cells is anti-inflammatory and protective, and iNKT cell-deficient $\mathrm{J} \alpha 18^{-/-}$mice had aggravated colitis and colon cancer [9,10], we suppose that the pro-inflammatory activities observed in our research was largely contributed by type II NKT cells, although the studies on the relationship of type II NKT cell and

neutrophils are still very limited. We think this mechanism is important because CD1d $\mathrm{d}^{-/-}$mice with both types of NKT cells eliminated had ameliorated colitis, indicating the pro-inflammatory effect of type II NKT cells outweighs $\underline{\text { the protective effect of iNKT cells in pathological conditions. Further investigation to characterize this subset may }}$ provide us with a way to specifically block the pro-inflammatory pathway while leaving the anti-inflammatory and anti-tumor iNKT subset intact.

\section{Acknowledgements}

This work was supported by the Major Research Plan of the National Natural Science Foundation of China (91229110), the National Science Foundation of China (81273215), the Specialized Research Fund for the Doctoral Program of Higher Education (20120071110046) and the Science and Technology Commission of Shanghai Municipality (13JC1407700),

\section{Conflicts of interest}

None

Fig 1. Colitis was ameliorated in CD1d ${ }^{-/}$mice. (A) The trends of weight change throughout the 6-day DSS treatment. (B) The relative body weights on Day 6. (C) The degree of splenomegaly on Day 6. (D) Gating splenic CD11b $\mathrm{b}^{+} \mathrm{Gr}-1^{\mathrm{hi}}$ neutrophils (left) and the percentage of neutrophils in splenocytes (right). (E) The proportion of TNF- $\alpha^{+}$neutrophils. (F)The histological sections of colons on Day 6 stained by hematoxylin \& eosin, representative specimens from each group are shown. Data of (A), (B), (C) and (D) are shown as mean \pm s.e.m. and are representative of more than 3 individual experiments. 
Fig 2 CD1d $^{-/-}$mice had decreased neutrophil recruitment and weaker neutrophil activities. (A) The gating strategy of lamina propria $\mathrm{CD} 11 \mathrm{~b}^{+} \mathrm{Gr}-1^{\mathrm{hi}}$ neutrophils (left) and their proportions in leukocytes (right). Representative of more than 3 individual experiments. (B) The gating strategy of TNF- $\alpha^{+}$neutrophils (left) and their proportions (right). (C) The mean fluorescence intensity (MFI) of DCF (a fluorochrome converted by cellular ROS from DCFH-DA) in neutrophils, representing ROS level. (D) The MFI of CXCR2 on neutrophils. (E) The expression of the neutrophil-attracting CXCL 1, 2, 3 and 5 in colon epithelial cells, analyzed using qPCR. In (B), (C), and (D), the WT+ $\mathrm{H}_{2} \mathrm{O}$ group have too few neutrophils and the data are not shown.

Fig 3. NKT cells strongly expressed TNF- $\alpha$ in lamina propria during colitis, and TNF- $\alpha$ could stimulate epithelial cells to secrete chemokines. (A) Gating of NKT cells (leftmost) and their TNF- $\alpha$ expressions (right). (B) The proportion of TNF- $\alpha^{+}$NKT cells during colitis. (C) The expression of CXCL 1, 2, 3 in epithelial cells isolated from wild type mice and incubated with indicated concentrations of TNF- $\alpha$ for indicated time, analyzed by qPCR. Three independent experiments showed similar trends.

Fig 4. Tumor load was relieved in CD1d $^{-/-}$mice in AOM-DSS model. (A) The schedule for tumor induction. (B) Comparison of tumor load in the colons between wild type and CD1 ${ }^{-/-}$mice. (C) The expression of cyclin D1 and beta-catenin in epithelium of colons analyzed using qPCR.

Fig. S1 CD11b ${ }^{+}$Gr-1 $^{\text {hi }}$ cells were FACS-sorted from the spleen and the colon lamina propria and stained with May-Grünwald-Giemsa dye.

\section{References:}

[1] J. Cosnes, C. Gower-Rousseau, P. Seksik, A. Cortot, Epidemiology and natural history of inflammatory bowel diseases, Gastroenterology, 140 (2011) 1785-1794.

[2] A.K.A. Vinay Kumar, Jon C. Aster, Robbins Basic Pathology, Ninth ed., Elsevier Saunders2013.

[3] A. Bendelac, P.B. Savage, L. Teyton, The biology of NKT cells, Annual review of immunology, 25 (2007) $297-336$.

[4] I.J. Fuss, F. Heller, M. Boirivant, F. Leon, M. Yoshida, S. Fichtner-Feigl, Z. Yang, M. Exley, A. Kitani, R.S. Blumberg, P. Mannon, W. Strober, Nonclassical CD1d-restricted NK T cells that produce IL-13 characterize an atypical Th2 response in ulcerative colitis, The Journal of clinical investigation, 113 (2004) 1490-1497. 
[5] I.J. Fuss, B. Joshi, Z. Yang, H. Degheidy, S. Fichtner-Feigl, H. de Souza, F. Rieder, F. Scaldaferri, A. Schirbel, M. Scarpa, G. West, C. Yi, L. Xu, P. Leland, M. Yao, P. Mannon, R.K. Puri, C. Fiocchi, W. Strober, IL-13Ralpha2-bearing, type II NKT cells reactive to sulfatide self-antigen populate the mucosa of ulcerative colitis, Gut, 63 (2014) 1728-1736. [6] F. Heller, I.J. Fuss, E.E. Nieuwenhuis, R.S. Blumberg, W. Strober, Oxazolone colitis, a Th2 colitis model resembling ulcerative colitis, is mediated by IL-13-producing NK-T cells, Immunity, 17 (2002) 629-638.

[7] A. Camelo, J.L. Barlow, L.F. Drynan, D.R. Neill, S.J. Ballantyne, S.H. Wong, R. Pannell, W. Gao, K. Wrigley, J. Sprenkle, A.N. McKenzie, Blocking IL-25 signalling protects against gut inflammation in a type-2 model of colitis by suppressing nuocyte and NKT derived IL-13, Journal of gastroenterology, 47 (2012) 1198-1211.

[8] C.M. Liao, M.I. Zimmer, S. Shanmuganad, H.T. Yu, S.L. Cardell, C.R. Wang, dysregulation of CD1d-restricted type ii natural killer T cells leads to spontaneous development of colitis in mice, Gastroenterology, 142 (2012) 326-334 e321-322. [9] H.S. Kim, D.H. Chung, IL-9-producing invariant NKT cells protect against DSS-induced colitis in an IL-4-dependent manner, Mucosal immunology, 6 (2013) 347-357.

[10] K. Yoshioka, Y. Ueno, S. Tanaka, K. Nagai, T. Onitake, R. Hanaoka, H. Watanabe, K. Chayama, Role of natural killer T cells in the mouse colitis-associated colon cancer model, Scandinavian journal of immunology, 75 (2012) 16-26.

[11] X. Liu, H. He, T. Huang, Z. Lei, F. Liu, G. An, T. Wen, Tanshinone IIA Protects against Dextran Sulfate Sodium(DSS-) Induced Colitis in Mice by Modulation of Neutrophil Infiltration and Activation, Oxidative medicine and cellular longevity, 2016 (2016) 7916763.

[12] S. Mathews, D. Feng, I. Maricic, C. Ju, V. Kumar, B. Gao, Invariant natural killer T cells contribute to chronic-plus-binge ethanol-mediated liver injury by promoting hepatic neutrophil infiltration, Cellular \& molecular immunology, (2015).

[13] L. Li, L. Huang, S.S. Sung, P.I. Lobo, M.G. Brown, R.K. Gregg, V.H. Engelhard, M.D. Okusa, NKT cell activation mediates neutrophil IFN-gamma production and renal ischemia-reperfusion injury, Journal of immunology, 178 (2007) 5899-5911.

[14] H. Wang, D. Feng, O. Park, S. Yin, B. Gao, Invariant NKT cell activation induces neutrophil accumulation and hepatitis: opposite regulation by IL-4 and IFN-gamma, Hepatology, 58 (2013) 1474-1485.

[15] C.C. Duwaerts, E.P. Sun, C.W. Cheng, N. van Rooijen, S.H. Gregory, Cross-activating invariant NKT cells and kupffer cells suppress cholestatic liver injury in a mouse model of biliary obstruction, PloS one, 8 (2013) e79702.

[16] S.J. Hwang, S. Kim, W.S. Park, D.H. Chung, IL-4-secreting NKT cells prevent hypersensitivity pneumonitis by suppressing IFN-gamma-producing neutrophils, Journal of immunology, 177 (2006) 5258-5268.

[17] M. Nakamatsu, N. Yamamoto, M. Hatta, C. Nakasone, T. Kinjo, K. Miyagi, K. Uezu, K. Nakamura, T. Nakayama, M. Taniguchi, Y. Iwakura, M. Kaku, J. Fujita, K. Kawakami, Role of interferon-gamma in Valpha14+ natural killer T cell-mediated host defense against Streptococcus pneumoniae infection in murine lungs, Microbes and infection / Institut Pasteur, 9 (2007) 364-374.

[18] R. Tang, G. Yang, S. Zhang, C. Wu, M. Chen, Opposite effects of interferon regulatory factor 1 and osteopontin on the apoptosis of epithelial cells induced by TNF-alpha in inflammatory bowel disease, Inflammatory bowel diseases, 20 (2014) 1950-1961.

[19] B. Yan, H. Wang, Z.N. Rabbani, Y. Zhao, W. Li, Y. Yuan, F. Li, M.W. Dewhirst, C.Y. Li, Tumor necrosis factor-alpha is a potent endogenous mutagen that promotes cellular transformation, Cancer research, 66 (2006) 11565-11570.

[20] L.B. Meira, J.M. Bugni, S.L. Green, C.W. Lee, B. Pang, D. Borenshtein, B.H. Rickman, A.B. Rogers, C.A. Moroski-Erkul, J.L. McFaline, D.B. Schauer, P.C. Dedon, J.G. Fox, L.D. Samson, DNA damage induced by chronic inflammation contributes to colon carcinogenesis in mice, The Journal of clinical investigation, 118 (2008) 2516-2525.

[21] G.B. Park, Y. Choi, Y.S. Kim, H.K. Lee, D. Kim, D.Y. Hur, ROS-mediated JNK/p38-MAPK activation regulates Bax translocation in Sorafenib-induced apoptosis of EBV-transformed B cells, International journal of oncology, 44 (2014) 977-985.

[22] G. Dent, S.C. Loweth, A.M. Hasan, F.M. Leslie, Synergic production of neutrophil chemotactic activity by colonic epithelial cells and eosinophils, Immunobiology, 219 (2014) 793-797. 
[23] J.H. Shin, S.H. Park, B Cells Promote Th1- Skewed NKT Cell Response by CD1d-TCR Interaction, Immune network, 13 (2013) 218-221.

[24] T.A. Ullman, S.H. Itzkowitz, Intestinal inflammation and cancer, Gastroenterology, 140 (2011) 1807-1816.

[25] S.I. Grivennikov, Inflammation and colorectal cancer: colitis-associated neoplasia, Seminars in immunopathology, 35 (2013) 229-244.

[26] K. Venken, T. Decruy, S. Aspeslagh, S. Van Calenbergh, B.N. Lambrecht, D. Elewaut, Bacterial CD1d-restricted glycolipids induce IL-10 production by human regulatory T cells upon cross-talk with invariant NKT cells, Journal of immunology, 191 (2013) 2174-2183.

[27] P.J. Brennan, R.V. Tatituri, M. Brigl, E.Y. Kim, A. Tuli, J.P. Sanderson, S.D. Gadola, F.F. Hsu, G.S. Besra, M.B. Brenner, Invariant natural killer $\mathrm{T}$ cells recognize lipid self antigen induced by microbial danger signals, Nature immunology, 12 (2011) 1202-1211.

[28] B.K. Popivanova, K. Kitamura, Y. Wu, T. Kondo, T. Kagaya, S. Kaneko, M. Oshima, C. Fujii, N. Mukaida, Blocking TNF-alpha in mice reduces colorectal carcinogenesis associated with chronic colitis, The Journal of clinical investigation, 118 (2008) 560-570.

[29] S. Yoshida, M. Ono, T. Shono, H. Izumi, T. Ishibashi, H. Suzuki, M. Kuwano, Involvement of interleukin-8, vascular endothelial growth factor, and basic fibroblast growth factor in tumor necrosis factor alpha-dependent angiogenesis, Molecular and cellular biology, 17 (1997) 4015-4023. 


\section{$\underset{A}{\mathrm{Fig}} 1$}

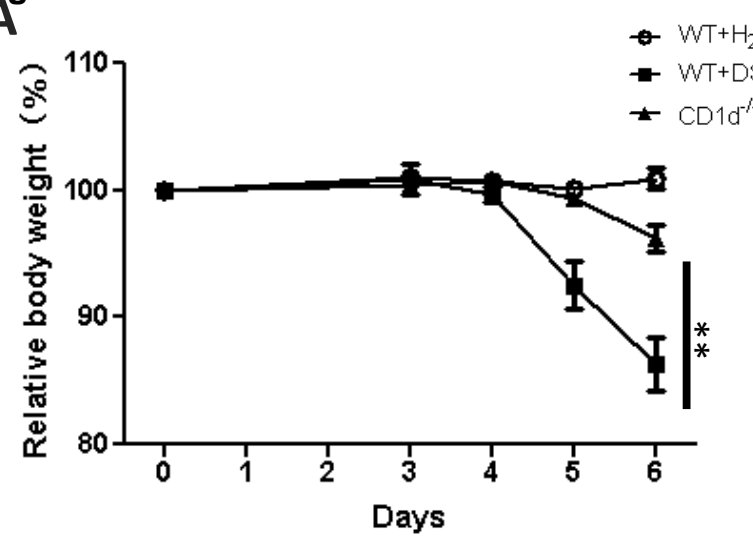

D

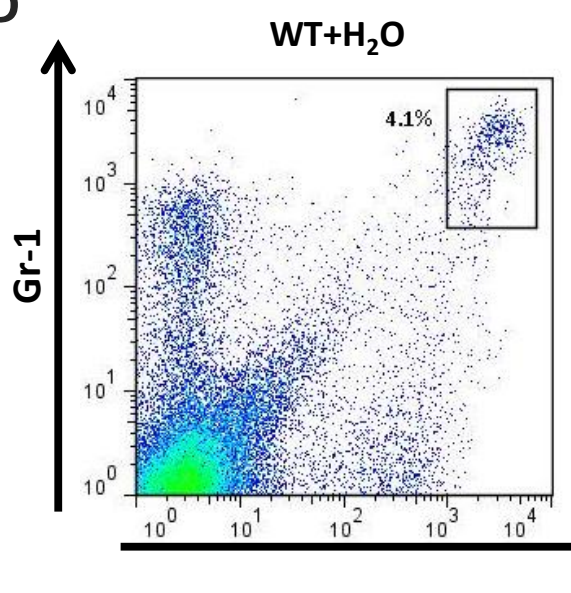

E

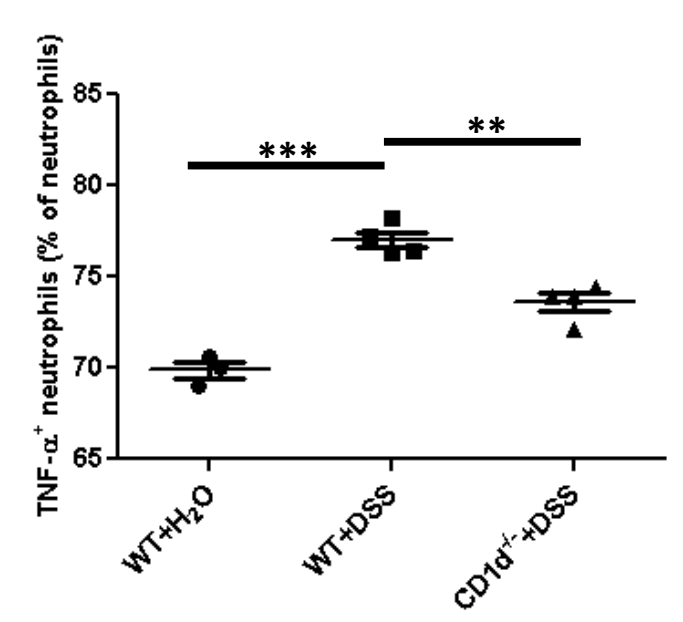

B
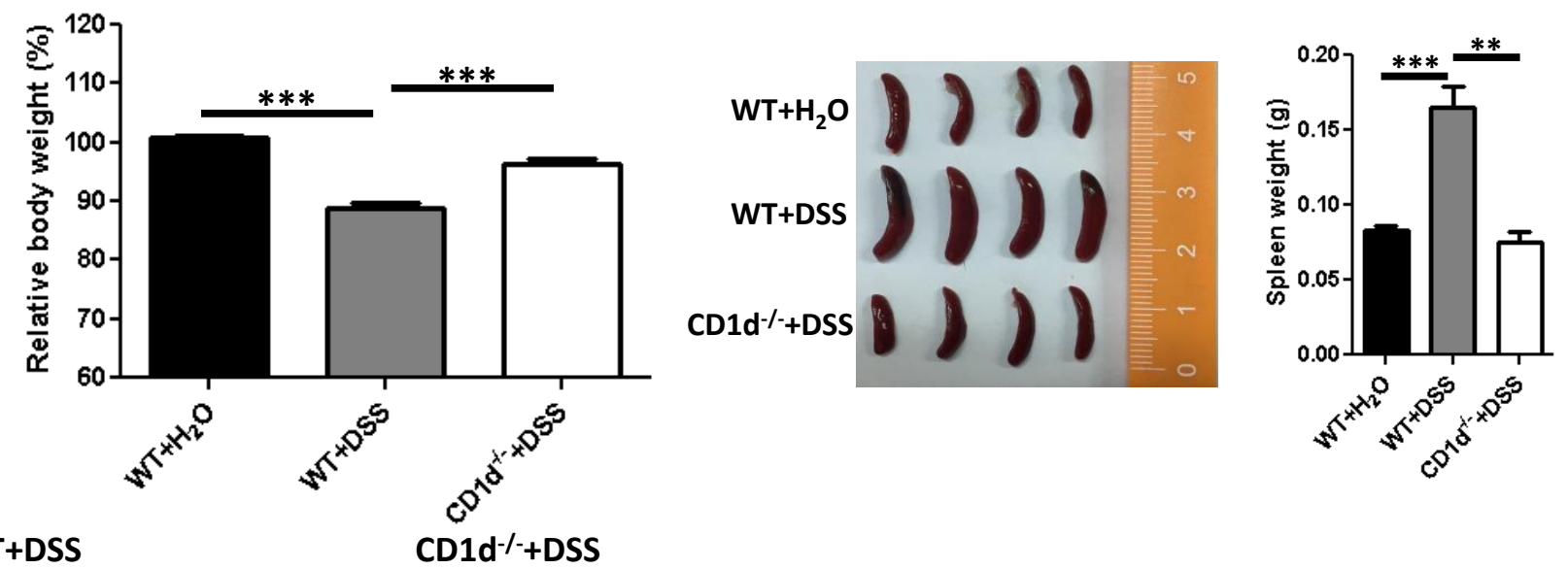

WT+DSS
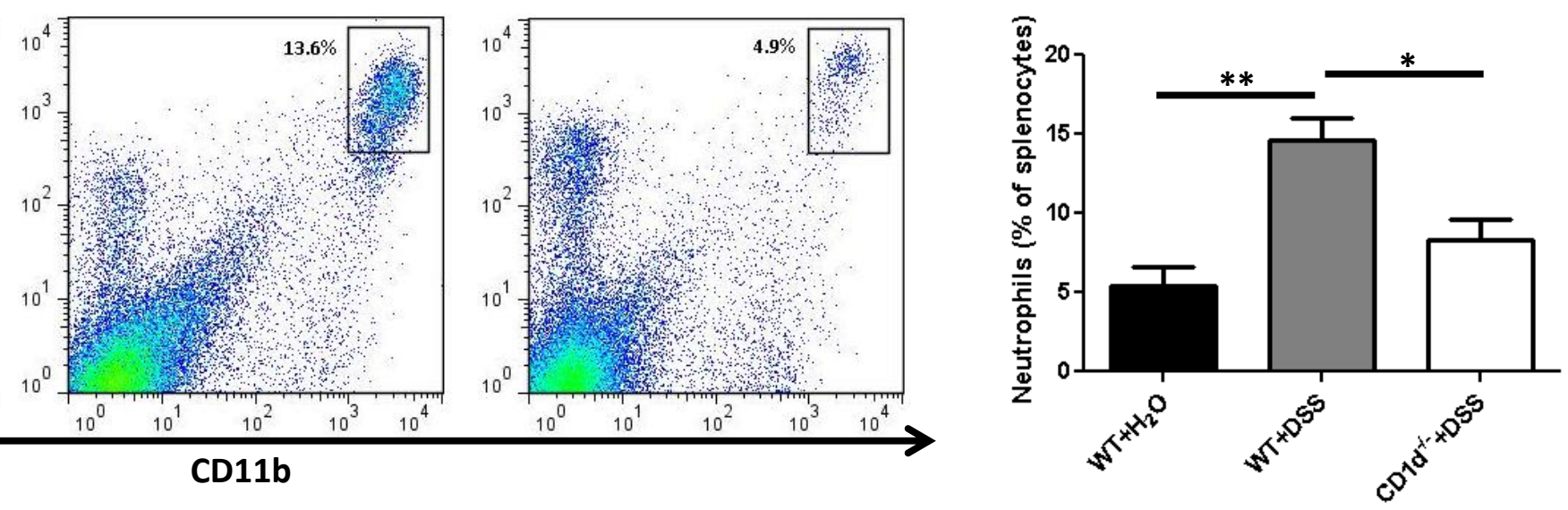

F

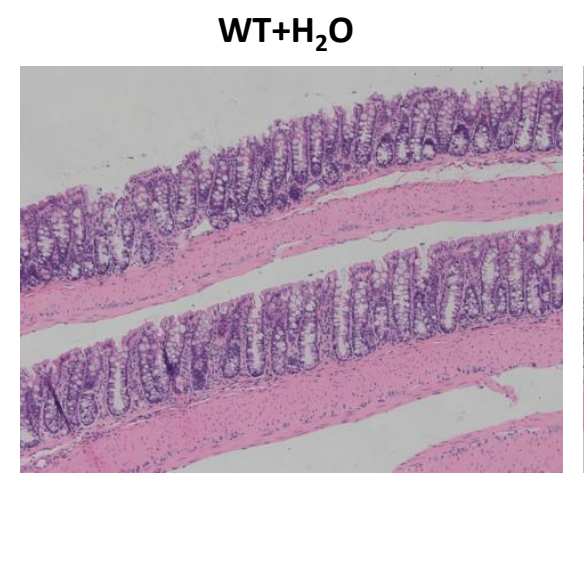

WT+DSS

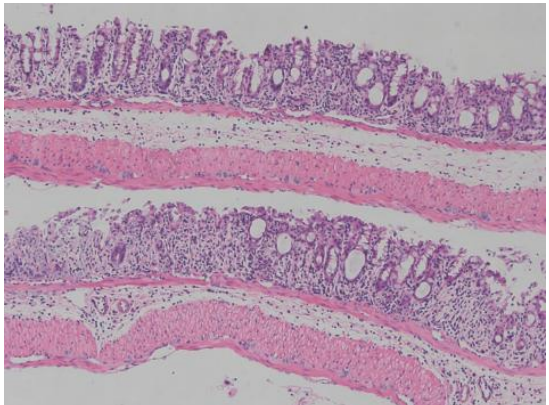

CD1d-/-DSS

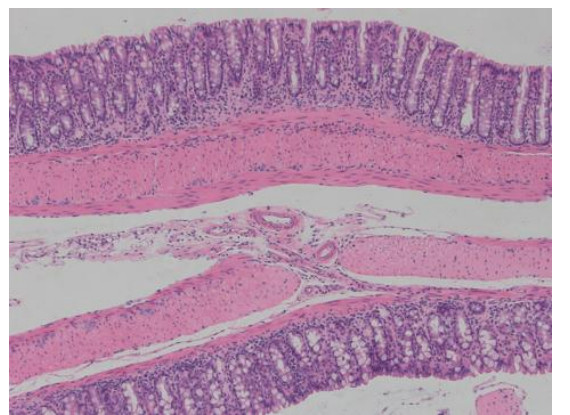


Fig 2
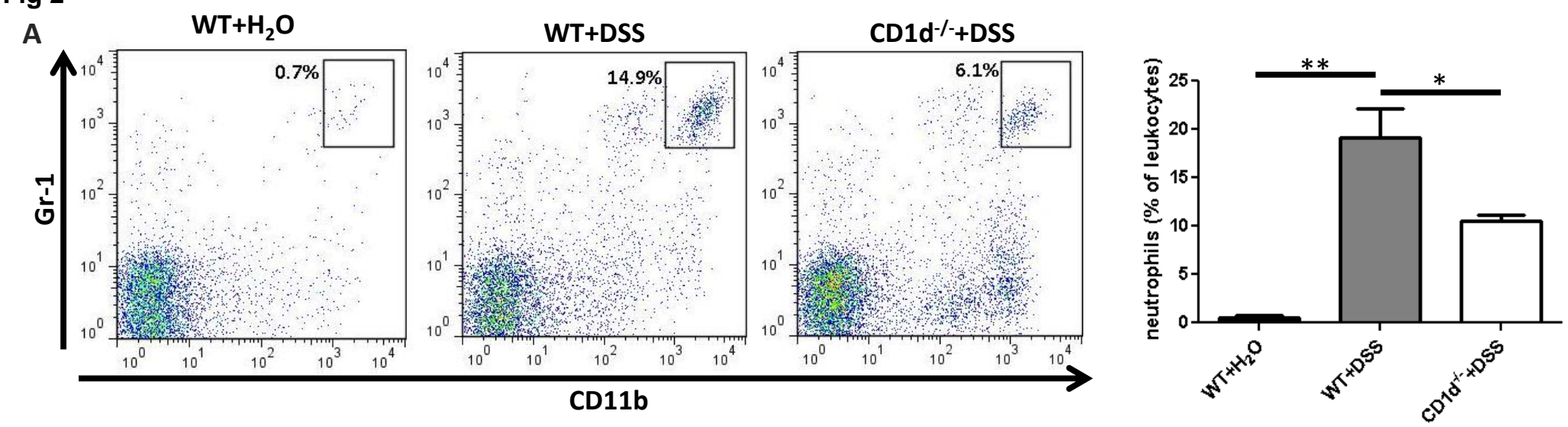

B
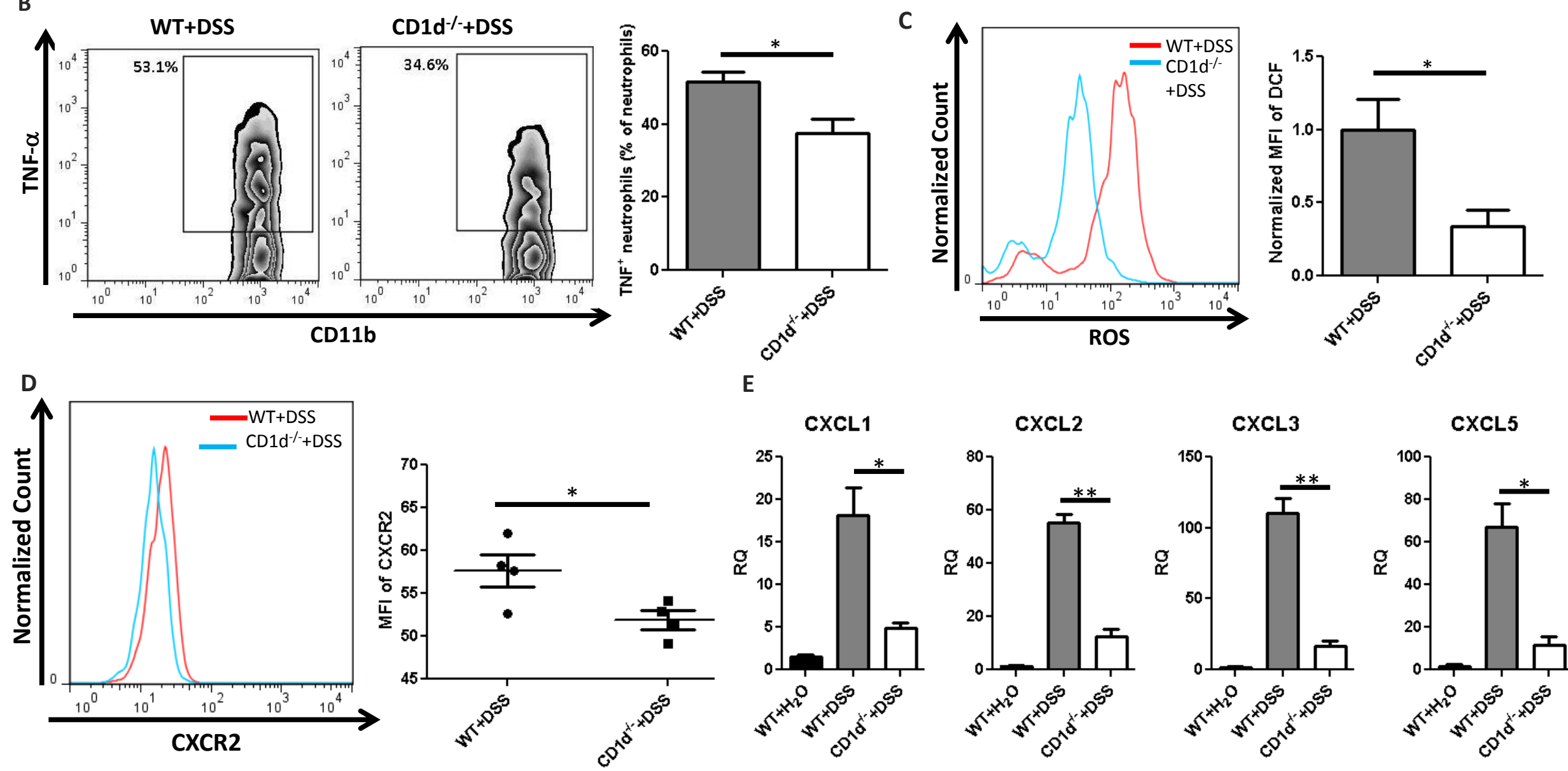

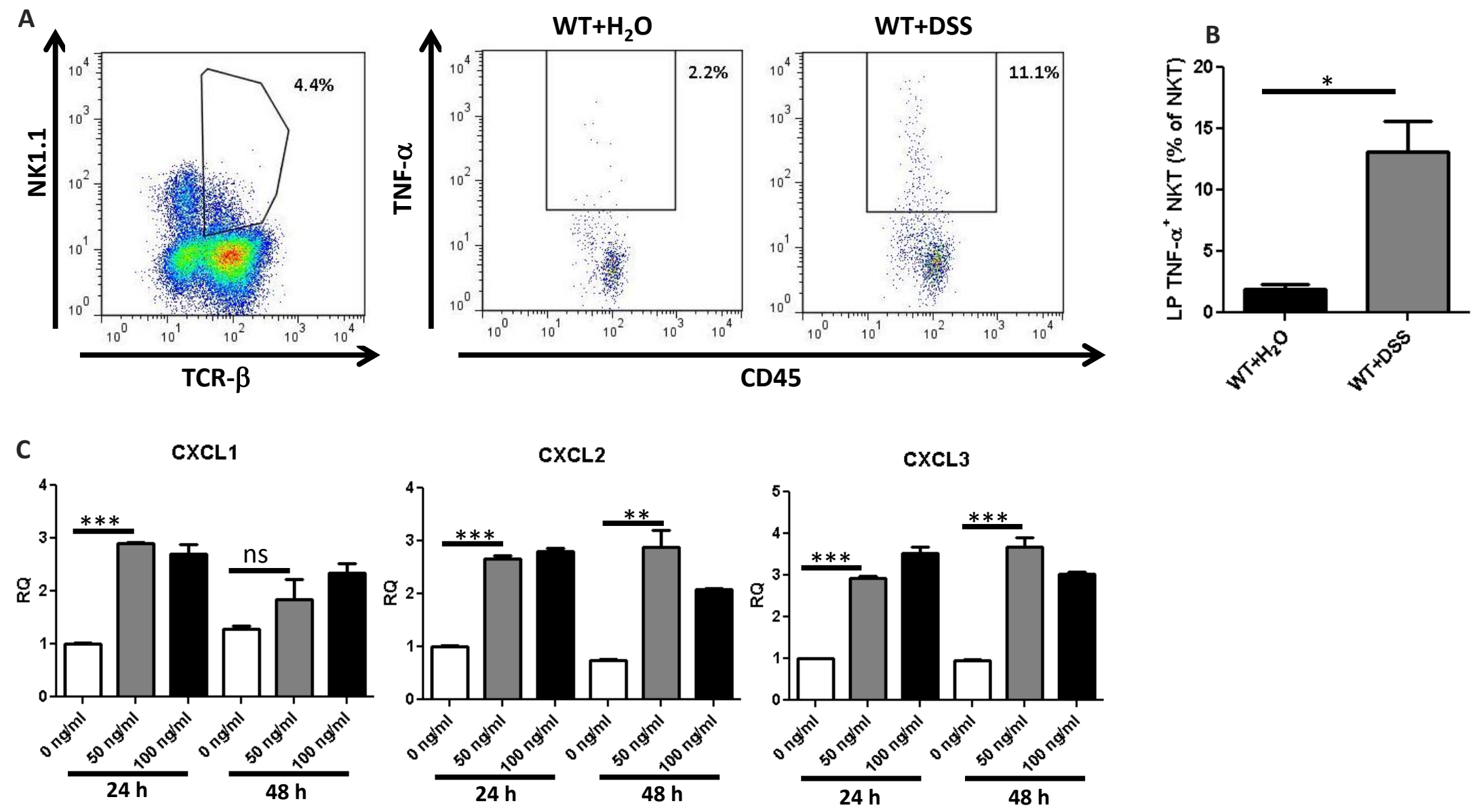
A

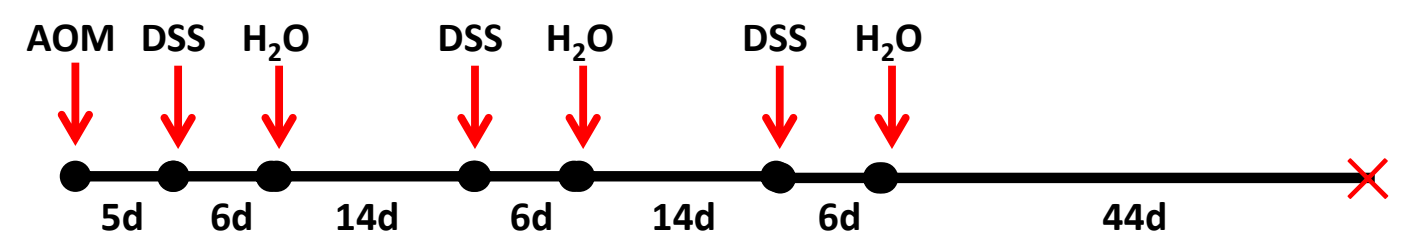

B

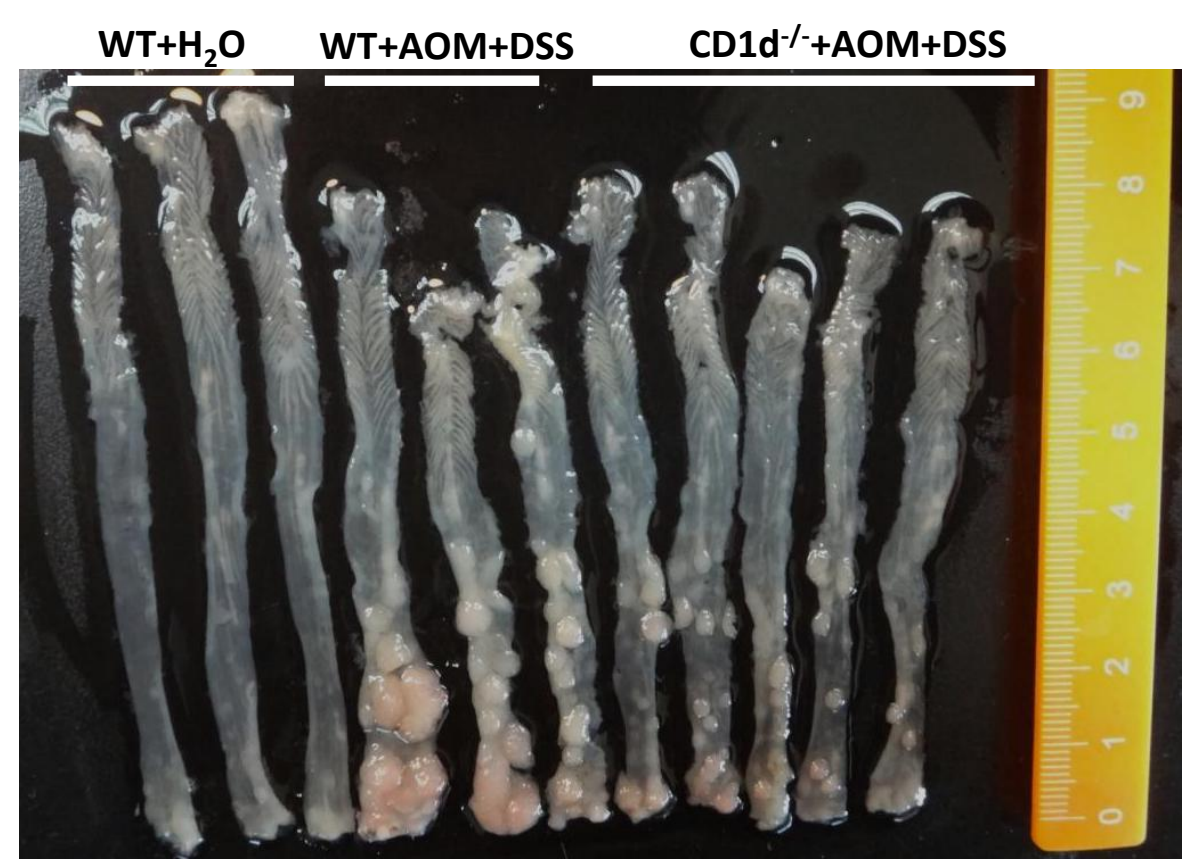

C
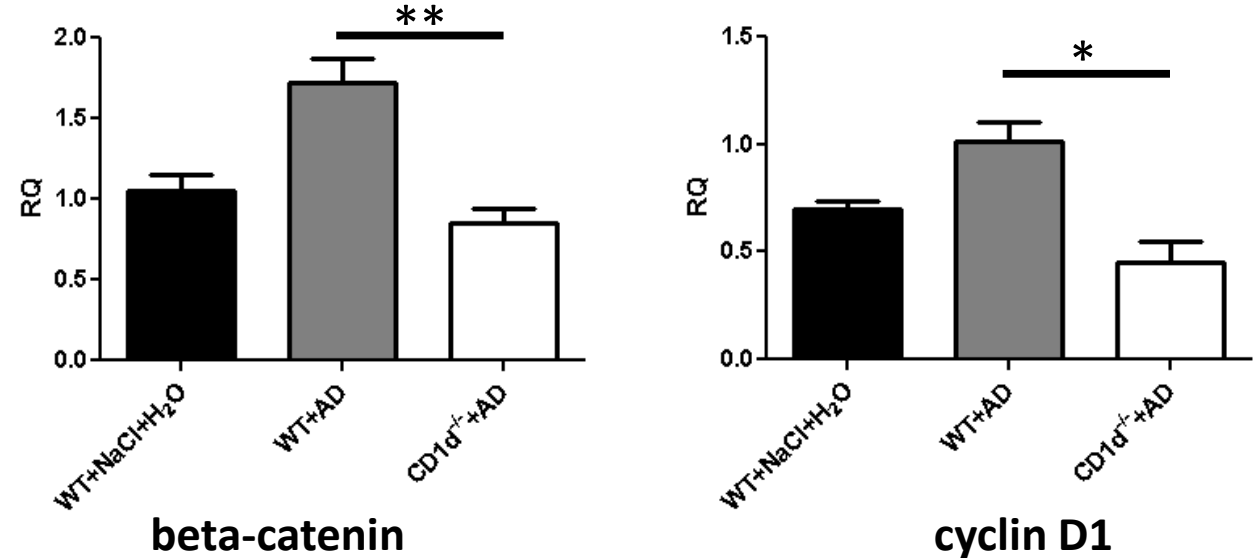